\title{
Asymptotic Behavior of Solutions to Diffusion Problems with Robin and Free Boundary Conditions
}

\author{
X. $\operatorname{Liu}^{1}$, B. $\operatorname{Lou}^{1 *}$ \\ ${ }^{1}$ Department of Mathematics, Tongji University, Shanghai 200092, China
}

\begin{abstract}
We study a nonlinear diffusion equation $u_{t}=u_{x x}+f(u)$ with Robin boundary condition at $x=0$ and with a free boundary condition at $x=h(t)$, where $h(t)>0$ is a moving boundary representing the expanding front in ecology models. For any $f \in C^{1}$ with $f(0)=0$, we prove that every bounded positive solution of this problem converges to a stationary one. As applications, we use this convergence result to study diffusion equations with monostable and combustion types of nonlinearities. We obtain dichotomy results and sharp thresholds for the asymptotic behavior of the solutions.
\end{abstract}

Keywords and phrases: Nonlinear diffusion equation, asymptotic behavior, Robin boundary condition, free boundary problem.

Mathematics Subject Classification: 35K20, 35K55, 35B40, 35R35

\section{Introduction}

The paper deals with the following nonlinear diffusion equation with Robin and free boundary conditions:

$$
\begin{cases}u_{t}=u_{x x}+f(u), & t>0,0<x<h(t), \\ u(t, 0)=b u_{x}(t, 0), & t>0, \\ u(t, h(t))=0, \quad h^{\prime}(t)=-\mu u_{x}(t, h(t)), & t>0, \\ h(0)=h_{0}, \quad u(0, x)=u_{0}(x), & 0 \leq x \leq h_{0},\end{cases}
$$

where $x=h(t)$ is a moving boundary to be determined together with $u(t, x), \mu>0$ and $b \geq 0$ are constants, $f$ satisfies

$$
f \in C^{1}([0, \infty)), \quad f(0)=0 \quad \text { and } \quad f(u) \leq K u(u \geq 0) \text { for some } K>0 .
$$

The initial function $u_{0}$ belongs to $\mathscr{X}\left(h_{0}\right)$ for some $h_{0}>0$, where

$$
\mathscr{X}\left(h_{0}\right):=\left\{\phi \in C^{2}\left(\left[0, h_{0}\right]\right): \phi(0)=b \phi^{\prime}(0), \phi\left(h_{0}\right)=0, \phi(x)>0 \text { in }\left(0, h_{0}\right)\right\} .
$$

In 2010, Du and Lin [4] studied the following problem:

$$
\begin{cases}u_{t}-d u_{x x}=u(1-u), & t>0,0<x<h(t), \\ u_{x}(t, 0)=0, & t>0, \\ u(t, h(t))=0, \quad h^{\prime}(t)=-\mu u_{x}(t, h(t)), & t>0, \\ h(0)=h_{0}, \quad u(0, x)=u_{0}(x), & 0 \leq x \leq h_{0},\end{cases}
$$

*Corresponding author. E-mail: blou@tongji.edu.cn 
They used this model to describe the spreading of a new or invasive species, with the free boundary $h(t)$ representing the expanding front. They proved a spreading-vanishing dichotomy result, namely the species either spreads to the whole environment and stabilizes at the positive steady state 1 , or it vanishes. They also gave a sharp threshold of $\mu$ which separate spreading phenomena ( $u$ converges to a positive steady state) and vanishing phenomena $(u \rightarrow 0)$. Their vanishing result improved the "hairtrigger effect" discovered in [2]. The latter says that any nonnegative solution of the Cauchy problem of $u_{t}=u_{x x}+u(1-u)$ converges to 1 as $t \rightarrow \infty$. It turns out that this conclusion does not match some biological observations. (For more biological backgrounds of the problems (1.3) and (1.1), we refer the readers to $[3-5,8]$ etc.).

In [7], the authors studied problem (1.1) with Dirichlet boundary condition at $x=0$ (i.e. $b=0$ ). For general nonlinearity $f$, they gave some sufficient conditions on the initial data for spreading. For the equation with logistic and cubic nonlinearity, they gave more sufficient conditions on spreading and vanishing.

In this paper we will study the free boundary problem (1.1) with a general nonlinearity $f(u)$ and with the Robin boundary condition at $x=0$. The Robin boundary condition includes the Dirichlet condition as a special case when $b=0$. (It does not includes the Neumann boundary condition since this case has been studied in [5]). Our main purpose is to give a convergence result for general $f$ as in [5], and then use it to study the asymptotic behavior for the solution of the equation with monostable and combustion types of nonlinearities.

Under the assumption (1.2), by a similar argument as in $[4,7]$ one can obtain the time-global existence for the solution $(u, h)$ of $(1.1)$ with $u \in C^{1+\nu / 2,2+\nu}((0, \infty) \times[0, h(t)])$ and $h \in C^{1+\nu / 2}((0, \infty))$ for any $\nu \in(0,1)$. By the maximum principle we have $u(t, x)>0$ for $t>0,0<x<h(t)$, and by the Hopf lemma we have $u_{x}(t, h(t))<0$ for $t>0$. Hence $h^{\prime}(t)>0$ and $h_{\infty}:=\lim _{t \rightarrow \infty} h(t) \in\left(h_{0}, \infty\right]$ exists. In what follows, denote

$$
I(t):=[0, h(t)) \quad \text { and } I_{\infty}:=\left[0, h_{\infty}\right) .
$$

We will show that any bounded nonnegative solution of (1.1) converges to a solution of the following elliptic problem:

$$
\left\{\begin{array}{l}
v^{\prime \prime}+f(v)=0, \quad x \in(0, \infty), \\
v(0)=b v^{\prime}(0)
\end{array}\right.
$$

Using a simple phase plane analysis (cf. $[2,5,6]$ ) one can see that, all the bounded nonnegative solutions of $(1.4)$ (defined in $[0, \infty)$ ) can be classified into the following categories:

- Type I. $v(x) \equiv 0$ for all $x \in[0, \infty)$;

- Type II. functions $v^{*}$ with $\left(v^{*}\right)^{\prime}(x)>0$ and $0 \leq v^{*}(x) \leq C$ for some $C>0$;

- Type III. $v(x)=\left.V\left(x-x_{0}\right)\right|_{[0, \infty)}$, where $x_{0}>0, V(x)$ is a even, positive solution of $(1.4)_{1}$ on $\mathbb{R}$ with $V^{\prime}(x)<0$ for $x>0$. A typical example of $V$ is the ground state when $f$ is a bistable type of nonlinearity;

- Type IV. $v(x)=\left.P(x)\right|_{[0, \infty)}$, where $P(x)>0$ is a periodic solution of $(1.4)_{1}$.

Our first main result is a general convergence theorem.

Theorem 1.1 (Convergence theorem). Assume that $f$ satisfy (1.2). Let $(u, h)$ be a solution of (1.1) defined for all $t>0$. If $u(t, x) \leq C$ for all $t>0$ and some $C>0$, then $u(t, \cdot)$ converges, as $t \rightarrow \infty$, locally uniformly in $[0, \infty)$ to a Type I, or Type II, or Type III solution of (1.4).

Recently, in [5] the authors studied the problem (1.1) with Robin boundary condition replaced by a Neumann one: $u_{x}(t, 0)=0$. They proved a similar convergence result as Theorem 1.1, and then applied it to equations with monostable, bistable and combustion types of nonlinearities. Among others, they obtained some dichotomy/trichotomy results on the asymptotic behavior of bounded solutions, obtained some sharp thresholds for spreading and vanishing. Their proof for the general convergence result depends essentially on the famous zero number properties as stated in [1] and [6]. Roughly speaking, these 
properties say that, for a solution of a linear parabolic equation, its degenerate zero lying in the interior of the domain makes the number of its zeros decreasing strictly. We also want to use such properties to prove Theorem 1.1. However, a linear parabolic equation corresponding to our problem (1.1) has a Robin boundary condition at $x=0$, and this end point might be a degenerate zero. So the results in [1] and [6] can not be applied directly. We will extend [1, Theorem $\mathrm{C}$ and $\mathrm{D}]$ to include the case that the end points can be degenerate zeros (see Lemmas 2.3 and 2.6 below for details). Such results will play a crucial role in our proof.

As applications of the general convergence theorem, we study two special cases:

$$
\left(\mathrm{f}_{M}\right) \text { monostable case, and }\left(\mathrm{f}_{C}\right) \text { combustion case. }
$$

In the monostable case $\left(\mathrm{f}_{M}\right)$, we assume that $f(u)$ satisfies $(1.2)$ and

$$
f(0)=f(1)=0, \quad f^{\prime}(0)>0, \quad f^{\prime}(1)<0, \quad(1-u) f(u)>0 \text { for } u>0, u \neq 1 .
$$

In the combustion case $\left(\mathrm{f}_{C}\right)$, we assume that $f(u)$ satisfies $(1.2)$ and for some $\theta \in(0,1)$,

$$
f(u)=0 \text { in }[0, \theta], \quad f(u)>0 \text { in }(\theta, 1), \quad f^{\prime}(1)<0, \quad f(u)<0 \text { for } u>1 .
$$

The following two theorems give a rather complete description of the asymptotic behavior of the solutions of (1.1) with these two types of nonlinearities.

Theorem 1.2 (The monostable case). Assume that $f$ is of $\left(\mathrm{f}_{M}\right)$ type, $u_{0} \in \mathscr{X}\left(h_{0}\right)$ for some $h_{0}>0$. Then either

(i) Spreading: $h_{\infty}=\infty$ and $\lim _{t \rightarrow \infty} u(t, x)=v^{*}(x)$ locally uniformly in $[0, \infty)$. Here $v^{*}$ is the unique Type II solution of (1.4), it increases strictly and converges to 1 as $x \rightarrow \infty$; or

(ii) Vanishing: $h_{\infty} \leq \frac{\pi}{\sqrt{f^{\prime}(0)}}$ and $\lim _{t \rightarrow \infty}\|u(t, \cdot)\|_{L^{\infty}(I(t))}=0$.

Moreover, if $h_{0}<\frac{\pi}{\sqrt{f^{\prime}(0)}}$, then there exists $\mu^{*} \geq 0$ depending on $u_{0}$, $h_{0}$ and $f$, such that vanishing happens when $\mu \leq \mu^{*}$ and spreading happens when $\mu>\mu^{*}$. In addition, $\mu^{*}>0$ when $h_{0}<\frac{\pi}{2 \sqrt{f^{\prime}(0)}}$ and $\left\|u_{0}\right\|_{L^{\infty}\left(\left[0, h_{0}\right]\right)}$ is sufficiently small.

Furthermore, for any $\phi \in \mathscr{X}\left(h_{0}\right)$ with $h_{0}<\frac{\pi}{\sqrt{f^{\prime}(0)}}$, if we consider the solution of (1.1) with initial data $u_{0}=\lambda \phi$, then there exists $\lambda^{*} \in[0, \infty]$, depending on $h_{0}, \phi$ and $f$, such that spreading happens when $\lambda>\lambda^{*}$ and vanishing happens when $\lambda \leq \lambda^{*}$.

Theorem 1.3 (The combustion case). Assume that $f$ is of $\left(\mathrm{f}_{C}\right)$ type, $u_{0} \in \mathscr{X}\left(h_{0}\right)$ for some $h_{0}>0$. Then either

(i) Spreading: $h_{\infty}=\infty$ and $\lim _{t \rightarrow \infty} u(t, x)=v^{*}(x)$ locally uniformly in $[0, \infty)$. Here $v^{*}$ is the unique Type II solution of (1.4), it increases strictly and converges to 1 as $x \rightarrow \infty$; or

(ii) Vanishing: $u(t, \cdot)$ converges to 0 locally uniformly in $I_{\infty}$ as $t \rightarrow \infty$.

Moreover, for any given $\phi \in \mathscr{X}\left(h_{0}\right)$, if we consider the solution $u$ of (1.1) with initial data $u_{0}=\lambda \phi$, then there exists $\lambda^{*} \in(0, \infty]$, depending on $h_{0}, \phi$ and $f$, such that spreading happens when $\lambda>\lambda^{*}$ and vanishing happens when $\lambda \leq \lambda^{*}$.

In [4] and [5], the authors even studied the asymptotic spreading speed when spreading happens. More precisely, they showed that, for each $\mu>0$,

$$
\left\{\begin{array}{l}
q^{\prime \prime}-c q^{\prime}+f(q)=0, \quad z>0 \\
q(0)=0, \mu q^{\prime}(0)=c, q(\infty)=1, q(z)>0 \text { for } z>0
\end{array}\right.
$$

has a unique solution $(c, q)=\left(c^{*}, q^{*}\right)$ with $c^{*}>0$, if $f$ is of $\left(\mathrm{f}_{M}\right)$ or $\left(\mathrm{f}_{C}\right)$ type. $q^{*}$ was called a "semi-wave" with speed $c^{*}$. Then they proved that the asymptotic spreading speed of $u$ equals $c^{*}$. For our problem (1.1) with $\left(\mathrm{f}_{M}\right)$ or $\left(\mathrm{f}_{C}\right)$ types of nonlinearity, we can obtain the same result:

$$
\lim _{t \rightarrow \infty} h(t) / t=c^{*} .
$$


Since the proof is similar as that in $[4,5]$, we omit the details in this paper.

When $f$ is a bistable type of nonlinearity, one can easily obtain some convergence results by Theorem 1.1. However, a complete description on the asymptotic behavior of solutions like those in Theorems 1.2 and 1.3 are much more complicated than the monostable and cumbustion cases. We will study it in a forthcoming paper.

In section 2 we give a proof for Theorem 1.1. In order to use the zero number argument, we first extend the famous zero number properties $[1$, Theorems $\mathrm{C}$ and $\mathrm{D}]$ to include the case that the end points might be degenerate zeros (Lemmas 2.3 and 2.6 below), then we use these key lemmas to prove Theorem 1.1. In section 3, we study monostable and combustion types $f$ as applications of Theorem 1.1. Theorem 1.2 and Theorem 1.3 follow from the results in subsections 3.1 and 3.2, respectively.

\section{General Convergence Theorem}

\subsection{Preliminaries}

We first prepare a comparison theorem which can be proved similarly as in [4, Lemma 3.5].

Lemma 2.1. Suppose that $\bar{h} \in C^{1}([0, T])$ for some $T>0$ and $\bar{u} \in C^{0,1}(\bar{\Omega}) \cap C^{1,2}(\Omega)$ with $\Omega=\{(t, x)$ : $0<t \leq T, 0<x<\bar{h}(t)\}$. Suppose also that

$$
\begin{cases}\bar{u}_{t} \geq \bar{u}_{x x}+f(\bar{u}), & 0<t \leq T, 0<x<\bar{h}(t), \\ \bar{u}(t, 0) \geq b \bar{u}_{x}(t, 0), & 0<t \leq T \\ \bar{u}(t, \bar{h}(t))=0, \quad \bar{h}^{\prime}(t) \geq-\mu \bar{u}_{x}(t, \bar{h}(t)), & 0<t \leq T .\end{cases}
$$

If $h_{0} \leq \bar{h}(0)$ and $u_{0}(x) \leq \bar{u}(0, x)$ on $\left[0, h_{0}\right]$, then the solution $(u, h)$ of $(1.1)$ satisfies:

$$
h(t) \leq \bar{h}(t) \text { in }(0, T], u(t, x) \leq \bar{u}(t, x) \text { for } t \in(0, T] \text { and } x \in[0, h(t)] .
$$

Remark 2.2. The pair $(\bar{u}, \bar{h})$ is usually called an upper solution of problem (1.1) and we can define a lower solution by reversing all the inequalities.

In what follows, for any continuous function $w$ defined on some interval $I=\left[x_{1}, x_{2}\right]$, we use $Z_{I}(w)$ to denote the number of zeros of $w$ on $I$. For convenience, we also use $I^{0}$ to denote its interior $\left(x_{1}, x_{2}\right)$. We now extend [1, Theorems $\mathrm{C}$ and $\mathrm{D}]$ to the case that allows the end points $x_{1}, x_{2}$ to be degenerate zeros.

Lemma 2.3. Let $I=\left[x_{1}, x_{2}\right]$. Assume that $w(t, x) \in C^{0,1}\left(\left[t_{1}, t_{2}\right] \times I\right) \cap C^{1,2}\left(\left(t_{1}, t_{2}\right) \times I^{0}\right)$ with $w(t, x) \not \equiv 0$ a solution of the equation

$$
w_{t}=w_{x x}+c(t, x) w, \quad t \in\left(t_{1}, t_{2}\right), x \in I^{0},
$$

where $c$ is a bounded function on $\left[t_{1}, t_{2}\right] \times I$. For $t \in\left(t_{1}, t_{2}\right)$, assume also that

$$
\text { (a) } \alpha_{i} w\left(t, x_{i}\right)+(-1)^{i}\left(1-\alpha_{i}\right) w_{x}\left(t, x_{i}\right)=0 \text { for some } \alpha_{i} \in[0,1], \quad \text { or } \quad(\mathrm{b}) \quad w\left(t, x_{i}\right) \neq 0,
$$

$i=1,2$. Then the following conclusions hold:

(i) $Z_{I}(w(t, \cdot))<\infty$ for any $t \in\left(t_{1}, t_{2}\right)$;

(ii) if $w\left(t^{*}, x^{*}\right)=w_{x}\left(t^{*}, x^{*}\right)=0$ for some $t^{*} \in\left(t_{1}, t_{2}\right), x^{*} \in I$, then

$$
Z_{I}(w(t, \cdot))>Z_{I}(w(s, \cdot)) \quad \text { for all } t \in\left(t_{1}, t^{*}\right), s \in\left(t^{*}, t_{2}\right) .
$$

Proof. We only prove the lemma in the case that $w$ satisfies (a) at $x=x_{1}$ for some $\alpha_{1} \in(0,1)$, and satisfies (b) at $x=x_{2}$ :

$$
w\left(t, x_{1}\right)=b w_{x}\left(t, x_{1}\right) \quad \text { and } \quad w\left(t, x_{2}\right) \neq 0 \quad \text { for } t \in\left(t_{1}, t_{2}\right),
$$


where $b=\frac{1-\alpha_{1}}{\alpha_{1}}>0$. Other cases can be proved similarly, some can also be found in [1]. Moreover, we write $x_{1}=0$ and $x_{2}=1$ for simplicity.

First, we claim that $w(t, 0) \not \equiv 0$ in any interval $\Delta \subset\left(t_{1}, t_{2}\right)$. Otherwise, we can extend $w$ to $\Delta \times(-1,0)$ as an odd function. Then (2.2) implies that $x=0$ is a degenerate zero of the extended function for all $t \in \Delta$. This contradicts the conclusions in [1, Theorems C and D]. Therefore, $\left\{t \in\left(t_{1}, t_{2}\right): w(t, 0)=0\right\}$ is a nowhere dense set. Thus there exists a time sequence $\left\{\tau_{n}\right\} \subset\left(t_{1}, t_{2}\right)$, decreasing to $t_{1}$, such that $w\left(\tau_{n}, 0\right) \neq 0$. For each $n$, we can find an $\varepsilon_{n}>0$ small such that

$$
w(t, 0) \neq 0 \quad \text { for } t \in\left(\tau_{n}-\varepsilon_{n}, \tau_{n}+\varepsilon_{n}\right) .
$$

Using [1, Theorem D] we have $Z_{I}(w(t, \cdot))<\infty$ for $t \in\left(\tau_{n}-\varepsilon_{n}, \tau_{n}+\varepsilon_{n}\right)$.

For any fixed $\tau_{n}$, we now study $Z_{I}(w(t, \cdot))$ for $t>\tau_{n}$. Denote $S_{n}:=\left\{t \in\left(\tau_{n}, t_{2}\right): w(t, 0)=0\right\}$. If $S_{n}=\emptyset$, then the conclusions of the lemma hold for $t \in S_{n}$ by [1, Theorem D]. Now we assume that $S_{n} \neq \emptyset$. Denote $\tilde{t}:=\inf S_{n} \in\left(\tau_{n}, t_{2}\right)$, then

$$
w(t, 0) \neq 0 \text { for } t \in\left(\tau_{n}, \tilde{t}\right) \quad \text { and } \quad 0=w(\tilde{t}, 0)\left(=b w_{x}(\tilde{t}, 0)\right) .
$$

By $\left[1\right.$, Theorem D], $Z_{I}(w(t, \cdot)) \leq Z_{I}\left(w\left(\tau_{n}, \cdot\right)\right)<\infty$ for $t \in\left(\tau_{n}, \tilde{t}\right)$. In $\left(\tau_{n}, \tilde{t}\right)$, since $w(t, \cdot)$ can have multiple zeros only at finite times, we may assume that $w(t, \cdot)$ has no multiple zeros when $t \in(\tilde{t}-\varepsilon, \tilde{t})$ for some $\varepsilon>0$. Assume further that the zeros of $w(t, \cdot)$ are

$$
0<x_{1}(t)<x_{2}(t)<\cdots<x_{k}(t)<1 \quad \text { for } t \in(\tilde{t}-\varepsilon, \tilde{t}) .
$$

By the continuity of $w$, for each $i=1,2, \cdots, k, x=x_{i}(t)$ is a continuous curve in the domain $(\tilde{t}-\varepsilon, \tilde{t}) \times$ $(0,1)$, extending to the line $t=\tilde{t}$. We now show that the zeros of $w(\tilde{t}, \cdot)$ are included in the set

$$
\tilde{S}:=\left\{0, \tilde{x}_{1}, \tilde{x}_{2}, \cdots, \tilde{x}_{k}\right\}
$$

where $\tilde{x}_{i}=x_{i}(\tilde{t}-0)(i=1,2, \cdots, k)$. Indeed, if there exists $\hat{x} \in\left(\tilde{x}_{i}, \tilde{x}_{i+1}\right)$ for some $0 \leq i \leq k$ $\left(\tilde{x}_{0}:=0, \tilde{x}_{k+1}:=1\right)$ such that $w(\tilde{t}, \hat{x})=0$, then we can use the maximum principle in the domain

$$
\left\{(t, x) \mid x_{i}(t)<x<x_{i+1}(t), \tilde{t}-\varepsilon<t \leq \tilde{t}\right\}
$$

to deduce a contradiction. Consequently, $Z_{I}(w(\tilde{t}, \cdot)) \leq k+1$. Therefore $x=0$ is an isolated zero of $w(\tilde{t}, \cdot)$. There exists $\tilde{x} \in(0,1)$ such that

$$
w(\tilde{t}, x) \neq 0 \quad \text { for } x \in(0, \tilde{x}] .
$$

By continuity of $w$, for any $\delta>0$ small we have

$$
w(t, \tilde{x}) \neq 0 \text { for } t \in[\tilde{t}-\delta, \tilde{t}+\delta] .
$$

If $w(\tilde{t}-\delta, x) \neq 0$ for $x \in[0, \tilde{x}]$, then we can use (2.4), (2.5) and the first inequality of (2.3) to conclude $w_{x}(\tilde{t}, 0) \neq 0$ by the Hopf lemma. This, however, contradicts the second equality of (2.3). Thus we have $Z_{[0, \tilde{x}]}(w(\tilde{t}-\delta, \cdot)) \geq 1$. On the other hand, combining (2.4), (2.5) with the boundary condition $w(t, 0)=b w_{x}(t, 0)$, we have $Z_{[0, \tilde{x}]}\left(w\left(\tilde{t}+\delta_{1}, \cdot\right)\right)=0$ by the maximum principle, for any small $\delta_{1}>0$.

Consequently, we obtain $Z_{[0, \tilde{x}]}\left(w\left(\tilde{t}+\delta_{1}, \cdot\right)\right)<Z_{[0, \tilde{x}]}(w(\tilde{t}-\delta, \cdot))$. By $(2.5)$, one also has $Z_{[\tilde{x}, 1]}(w(\tilde{t}+$ $\left.\left.\delta_{1}, \cdot\right)\right)<Z_{[\tilde{x}, 1]}(w(\tilde{t}-\delta, \cdot))$ by $\left[1\right.$, Theorem D]. Therefore, we get $Z_{I}\left(w\left(\tilde{t}+\delta_{1}, \cdot\right)\right)<Z_{I}(w(\tilde{t}-\delta, \cdot))$.

Since $\tau_{n}$ can be chosen as close as possible to $t_{1}$, we prove the lemma.

Remark 2.4. This lemma shows that the number of zeros of $w$ decreases strictly at the time when it has a degenerate zero, even if the degenerate zero is an end point (provided it satisfies boundary condition (a)). 
Corollary 2.5. If $w$ satisfies all the assumptions in Lemma 2.3 for $t_{2}=\infty$, then there exists $T>t_{1}$ such that $w(t, \cdot)$ has no degenerate zeros (including $x_{1}$ and $x_{2}$ ) for $t>T$.

Lemma 2.6. Let $w_{n}(t, x)$ be a sequence of functions converging to $w(t, x)$ in $C^{0,1}\left(\left[t_{1}, t_{2}\right] \times\left[x_{1}, x_{2}\right]\right)$. Assume that, for each $t \in\left[t_{1}, t_{2}\right]$ and $n \in \mathbb{N}$, the function $w_{n}(t, \cdot)$ has only simple zeros on $\left[x_{1}, x_{2}\right]$. Assume also that $w(t, x)$ satisfies an equation of the form $(2.1)$ on $\left[t_{1}, t_{2}\right] \times\left[x_{1}, x_{2}\right]$, and $w$ satisfies condition (a) or (b) in Lemma 2.3 at the end points. Then for each $t \in\left[t_{1}, t_{2}\right]$, either $w(t, x) \equiv 0$ on $\left[x_{1}, x_{2}\right]$, or $w(t, x)$ has only simple zeros on $\left[x_{1}, x_{2}\right]$.

The proof for this lemma is similar as that for Lemma 2.6 in [6]. Indeed, our lemma can be regarded as a complementarity of [6, Lemma 2.6], since we consider not only degenerate zeros in $\left(x_{1}, x_{2}\right)$, but also the degeneracy of the end points $x_{1}$ and $x_{2}$.

\subsection{Proof of Theorem 1.1}

In this subsection assume that all the assumptions in Theorem 1.1 hold, and then prove this general convergence result. The proof is divided into several lemmas.

Denote by $\omega(u)$ the $\omega$-limit set of $u(t, \cdot)$ in the topology of $L_{l o c}^{\infty}\left(I_{\infty}\right)$. In other words, a function $w(x)$ belongs to $\omega(u)$ if and only if there exists a sequence $0<t_{1}<t_{2}<t_{3}<\cdots \rightarrow \infty$ such that

$$
\lim _{n \rightarrow \infty} u\left(t_{n}, x\right)=w(x) \text { locally uniformly in } I_{\infty} .
$$

By local parabolic estimates, we see that the convergence (2.6) implies convergence in the $C_{\text {loc }}^{2}\left(I_{\infty}\right)$ topology. Thus the definition of $\omega(u)$ remains unchanged if the topology of $L_{l o c}^{\infty}\left(I_{\infty}\right)$ is replaced by that of $C_{l o c}^{2}\left(I_{\infty}\right)$.

It is well-known that $\omega(u)$ is a compact, connected and invariant set. This means that for any $w \in \omega(u)$ there exists an entire orbit (namely a solution of $W_{t}=W_{x x}+f(W)$ defined for all $t \in \mathbb{R}$ and $x \in I_{\infty}$ ) passing through $w$. Choosing a suitable sequence $0<t_{1}<t_{2}<t_{3}<\cdots \rightarrow \infty$, we can find such an entire solution $W(t, x)$ with $W(0, x)=w(x)$ and

$$
u\left(t+t_{n}, x\right) \rightarrow W(t, x) \quad \text { as } n \rightarrow \infty .
$$

Here the convergence is understood in the topology of $L_{l o c}^{\infty}\left(\mathbb{R} \times I_{\infty}\right)$, but, by parabolic regularity, it takes place in the $C_{l o c}^{1,2}\left(\mathbb{R} \times I_{\infty}\right)$ sense.

Lemma 2.7. $\omega(u)$ consists of bounded, nonnegative solutions of $v^{\prime \prime}+f(v)=0\left(x \in I_{\infty}\right)$.

Proof. Let $w$ be an arbitrary element of $\omega(u)$ and $W(t, x)$ be the entire orbit satisfying $W(0, x)=w(x)$ as given in (2.7). Let $v(x)$ be the unique solution of

$$
v^{\prime \prime}+f(v)=0 \quad \text { with } \quad v(0)=w(0) \text { and } v^{\prime}(0)=w^{\prime}(0) .
$$

Since $w(x)$, as one $\omega$-limit point of $u$, satisfies $w(0)=b w^{\prime}(0) \geq 0$, we have $w(0)=w^{\prime}(0)=0$, or $w(0)=0$, $w^{\prime}(0)>0$ (only when $b=0$ ), or $w(0)>0, w^{\prime}(0)>0$ (only when $b>0$ ). Therefore, $v$ is either 0 , or a positive solution of $(2.8)$ in $(0, \infty)$, or a solution of $(2.8)$ with compact positive support. The last one means that

$$
v(x)>0 \text { in }\left(0, R_{0}\right), \quad v\left(R_{0}\right)=0 \text { or } v\left(R_{0}-0\right)=\infty,
$$

for some $R_{0} \in(0, \infty)$. We may now use Lemmas 2.3 and 2.6 and follow the argument in the proof of Lemma 3.4 in [6] (with obvious minor variations) to conclude that $w \equiv v$. This proves the lemma.

Lemma 2.8. $\omega(u)$ is a singleton. 
Proof. Let $v_{1}, v_{2}$ be two elements in $\omega(u)$. We only need to show that $v_{1}(0)=v_{2}(0)$ and $v_{1}^{\prime}(0)=v_{2}^{\prime}(0)$ since this implies $v_{1} \equiv v_{2}$ by $(2.8)$. Suppose that $v_{1}(0) \neq v_{2}(0)$ and we will derive a contradiction (the case where $v_{1}(0)=v_{2}(0)$ and $v_{1}^{\prime}(0) \neq v_{2}^{\prime}(0)$ is discussed similarly). Without loss of generality we may assume that $v_{1}(0)<\gamma<v_{2}(0)$ for some constant $\gamma$. Since $\omega(u)$ is a connected set, there exists $w \in \omega(u)$ such that $w(0)=\gamma$. Moreover, by Lemma 2.7, $w$ is a nonnegative solution of $v^{\prime \prime}+f(v)=0\left(x \in I_{\infty}\right)$.

Set $\eta(t, x):=u(t, x)-w(x)$. Then $\eta$ satisfies

$$
\eta_{t}=\eta_{x x}+c(t, x) \eta, \quad t>0, x \in I(t)
$$

for some bounded function $c$. By zero number property, we have $Z_{I(t)}(\eta(t, \cdot))<\infty$.

On the other hand, there exists a sequence $t_{n}^{(i)} \nearrow \infty(i=1,2)$ such that $u\left(t_{n}^{(i)}, x\right) \rightarrow v_{i}(x)$ as $n \rightarrow \infty$. Hence for large $n, \eta\left(t_{n}^{(1)}, 0\right)<0$ and $\eta\left(t_{n}^{(2)}, 0\right)>0$. So $\eta(t, 0)$ changes sign infinitely many times. This is impossible by $\eta(t, 0)=b \eta_{x}(t, 0)$ and Lemma 2.3. The lemma is proved.

Lemma 2.9. Let $b>0$ and $p$ be a positive periodic solution of (1.4). Then problem (1.4) has another periodic solution $\tilde{p}$ such that

$$
\tilde{p}_{+}:=\max _{x \geq 0} \tilde{p}(x)>p_{+}:=\max _{x \geq 0} p(x), \quad 0<\tilde{p}_{-}:=\min _{x \geq 0} \tilde{p}(x)<p_{-}:=\min _{x \geq 0} p(x) .
$$

Proof. It is easily seen that any nonnegative solution of $v^{\prime \prime}+f(v)=0$ is symmetric with respect to each critical point. Hence if

$$
p\left(x_{0}\right)=p_{-}, \quad p\left(x_{0}+\ell\right)=p_{+} \quad \text { and } \quad p^{\prime}(x)>0\left(x_{0}<x<x_{0}+\ell\right),
$$

for some $x_{0} \geq 0$, then $p\left(x_{0}+2 \ell\right)=p_{-}$and $2 \ell$ is the period of $p$. Since $p$ satisfies

$$
p^{\prime \prime}+f(p)=0 \quad \text { and } \quad p\left(x_{0}+\ell\right)=p_{+}, \quad p^{\prime}\left(x_{0}+\ell\right)=0,
$$

by the uniqueness of solution of this initial value problem we know that $f\left(p\left(x_{0}+\ell\right)\right)=f\left(p_{+}\right)>0$. Similarly, we have $f\left(p_{-}\right)<0$. Therefore, there exists $\delta_{1}>0$ such that

$$
f\left(p_{+}+\delta\right)>0 \text { for any } \delta \in\left[0, \delta_{1}\right) .
$$

For $\delta_{2} \leq \frac{1}{6}\left(p_{+}-p_{-}\right)$, we choose $x_{1}, x_{2}$ satisfying $x_{0}<x_{1}<x_{0}+\ell<x_{2}<x_{0}+2 \ell$ such that $p\left(x_{1}\right), p\left(x_{2}\right)=p_{+}-2 \delta_{2}$.

Now we consider the initial value problem

$$
v^{\prime \prime}+f(v)=0 \quad(x \geq 0) \quad \text { with } \quad v\left(x_{0}\right)=p_{-}-\frac{1}{n}, \quad v^{\prime}\left(x_{0}\right)=0 .
$$

For each positive integer $n$, denote its solution by $v_{n}(x)$. For sufficiently large $n$ we have

$$
\left|v_{n}(x)-p(x)\right|<\min \left\{\delta_{1}, \delta_{2}\right\} \quad \text { for } x \in\left[x_{0}, x_{0}+2 \ell\right] .
$$

Hence at $x_{i}(i=1,2)$ we have

$$
v_{n}\left(x_{i}\right)<p\left(x_{i}\right)+\delta_{2}=p\left(x_{0}+\ell\right)-\delta_{2}<v_{n}\left(x_{0}+\ell\right) .
$$

This implies that $v_{n}^{\prime}\left(y_{n}\right)=0$ at some $y_{n} \in\left(x_{1}, x_{2}\right)$. Similarly, one can find another critical point near $x_{0}+2 \ell$. Hence $v_{n}$ is a periodic function.

Next we prove (2.9). Multiplying the equation of $v_{n}$ by $v_{n}^{\prime}$ and integrating it on $\left[x_{0}, y_{n}\right]$ we have

$$
0=\int_{x_{0}}^{y_{n}} f\left(v_{n}(x)\right) v_{n}^{\prime}(x) d x=\int_{p_{-} \frac{1}{n}}^{v_{n}\left(y_{n}\right)} f(s) d s=\left(\int_{p_{-}-\frac{1}{n}}^{p_{-}}+\int_{p_{-}}^{p_{+}}+\int_{p_{+}}^{v_{n}\left(y_{n}\right)}\right) f(s) d s .
$$


When $n$ is large, the first integral is negative since $f\left(p_{-}\right)<0$, the second integral is exactly 0 , and so the third integral is positive. Combining with (2.10) and (2.11) we see that $v_{n}\left(y_{n}\right)>p_{+}$. This proves (2.9).

Finally, we show that a suitable shift of $v_{n}$ satisfies the Robin boundary condition in (1.4). Consider the function

$$
F(r):=\frac{r^{2}}{b^{2}}+2 \int_{p_{-}-\frac{1}{n}}^{r} f(s) d s \text { for } r \in\left[p_{-}-\frac{1}{n}, \infty\right) .
$$

We show that $F\left(r_{1}\right)<0$ for some $r_{1} \in\left(p_{-}-\frac{1}{n}, p_{+}\right)$. Since $p$ satisfies the Robin boundary condition, there exists $x_{3} \in\left(x_{0}, x_{0}+\ell\right)$ such that $p\left(x_{3}\right)=b p^{\prime}\left(x_{3}\right)$. Multiplying the equation of $p$ by $p^{\prime}$ and integrating it on $\left[x_{0}, x_{3}\right]$ we have

$$
\left[p^{\prime}\left(x_{3}\right)\right]^{2}+2 \int_{p_{-}}^{p\left(x_{3}\right)} f(s) d s=0 .
$$

Therefore, for $r_{1}:=p\left(x_{3}\right)$ we have

$$
F\left(r_{1}\right)=\left(\frac{p\left(x_{3}\right)}{b}\right)^{2}+2\left(\int_{p_{-}-\frac{1}{n}}^{p_{-}}+\int_{p_{-}}^{p\left(x_{3}\right)}\right) f(s) d s=2 \int_{p_{-}-\frac{1}{n}}^{p_{-}} f(s) d s<0,
$$

provided $n$ is large. On the other hand, $F\left(p_{-}-\frac{1}{n}\right)>0$. So $F\left(r_{2}\right)=0$ for some $r_{2} \in\left(p_{-}-\frac{1}{n}, r_{1}\right)$. Assume that $v_{n}(\bar{x})=r_{2}$ for some $\bar{x} \in\left(x_{0}, y_{n}\right)$, then

$$
\frac{v_{n}^{2}(\bar{x})}{b^{2}}+2 \int_{p_{--\frac{1}{n}}}^{v_{n}(\bar{x})} f(s) d s=F\left(r_{2}\right)=0=\left[v_{n}^{\prime}(\bar{x})\right]^{2}+2 \int_{p_{-}-\frac{1}{n}}^{v_{n}(\bar{x})} f(s) d s .
$$

The last equality is obtained by multiplying the equation of $v_{n}$ by $v_{n}^{\prime}$ and integrating it on $\left[x_{0}, \bar{x}\right]$. Hence $v_{n}(\bar{x})=b v_{n}^{\prime}(\bar{x})$ and so $v(x):=v_{n}(x+\bar{x})$ satisfies the Robin boundary condition in (1.4).

Using this lemma we can prove that no periodic solution can be an $\omega$-limit of $u$.

Lemma 2.10. Let $v$ be an element of $\omega(u)$. Then $v(x)$ can not be a positive periodic solution of (1.4).

Proof. Suppose, on the contrary, that $\omega(u)=\{p(x)\}$. By Lemma 2.9 problem (1.4) has another positive periodic solution $\tilde{p}(x)$ such that

$$
\max \tilde{p}(x)>\max p(x)>\min p(x)>\min \tilde{p}(x) .
$$

Then $\xi(t, x):=u(t, x)-\tilde{p}(x)$ satisfies

$$
\begin{cases}\xi_{t}=\xi_{x x}+c(t, x) \xi, & x \in(0, h(t)), t>0 \\ \xi(t, 0)=b \xi_{x}(t, 0), \quad \xi(t, h(t))<0, & t>0\end{cases}
$$

for some bounded function $c$. By zero number property Lemma 2.3, we have $Z_{[0, h(t)]}(\xi(t, \cdot))<\infty$ and it is nonincreasing in $t$. On the other hand, by our assumption $\omega(u)=\{p(x)\}, \xi(t, x) \rightarrow p(x)-\tilde{p}(x)$ as $t \rightarrow \infty$. So $Z_{[0, h(t)]}(\xi(t, \cdot)) \rightarrow \infty$ by $(2.12)$. This contradiction proves the lemma.

Lemma 2.11. If $h_{\infty}<\infty$, then $\omega(u)=\{0\}$ and $\lim _{t \rightarrow \infty}\|u(t, \cdot)\|_{L^{\infty}(I(t))}=0$.

Proof. The lemma can be proved in a similar way as in $[4,5]$ by using $L^{p}$ estimates and Sobolev embeddings, or as in [7] by using semigroup theory. Here we give an elementary proof, which only depends on the construction of simple upper solutions.

By Lemmas 2.7 and $2.8, u(t, \cdot)$ converges locally uniformly to a solution $v$ of

$$
v^{\prime \prime}+f(v)=0 \quad \text { for } x \in I_{\infty} .
$$


Note that we yet do not know what $v\left(h_{\infty}-0\right)$ is. Now we show that $v\left(h_{\infty}-0\right)$ is indeed 0 .

By the assumptions in Theorem 1.1, $u(t, x) \leq C$ for some $C>0$. Set

$$
\Omega_{M}:=\left\{(t, x): t>0, h(t)-M^{-1} \leq x \leq h(t)\right\},
$$

and

$$
w(t, x):=C\left[2 M(h(t)-x)-M^{2}(h(t)-x)^{2}\right],
$$

where

$$
M \geq \max \left\{\sqrt{\frac{K}{2}}, \frac{4\left\|u_{0}\right\|_{C^{1}\left(\left[0, h_{0}\right]\right)}}{3 C}\right\},
$$

$K$ is that in (1.2). As in the proof of [4, Lemma 2.2] one can show that

$$
u(t, x) \leq w(t, x) \quad \text { in } \Omega_{M} .
$$

For any given $\varepsilon>0$, take $\delta:=\min \left\{\frac{\varepsilon}{2 M C}, \frac{1}{M}\right\}$. Then there exists $T>0$ such that

$$
h_{\infty}-\frac{1}{M} \leq h_{\infty}-\delta<h(t)<h_{\infty} \text { for } t>T
$$

Thus, for $t>T$ and $x \in\left[h_{\infty}-\delta, h(t)\right]$ we have

$$
u(t, x) \leq w(t, x) \leq w\left(t, h_{\infty}-\delta\right) \leq \lim _{t \rightarrow \infty} w\left(t, h_{\infty}-\delta\right)=C\left(2 M \delta-M^{2} \delta^{2}\right)<\varepsilon .
$$

Therefore, for any $\bar{x} \in\left[h_{\infty}-\delta, h_{\infty}\right), u(t, \bar{x})<\varepsilon$ for sufficiently large $t$, and so $v(\bar{x}) \leq \varepsilon$. Since $\varepsilon \rightarrow 0$ as $\delta \rightarrow 0$, we indeed have $v\left(h_{\infty}-0\right)=0$.

Defining $v\left(h_{\infty}\right)=0$ and combining with (2.13) we see that $v$ is indeed a solution of the following two-point boundary value problem of elliptic equation:

$$
\left\{\begin{array}{l}
v^{\prime \prime}+f(v)=0, \quad 0<x<h_{\infty}<\infty \\
v(0)=b v^{\prime}(0) \geq 0, \quad v\left(h_{\infty}\right)=0
\end{array}\right.
$$

If $v \not \equiv 0$, then by $v \geq 0$ and the Hopf lemma we have

$$
v\left(h_{\infty}\right)=0, \quad v^{\prime}\left(h_{\infty}\right)<0
$$

We will derive a contradiction from this.

For each $n \in \mathbb{N}$, we set $v_{n}:=\max \left\{v-\frac{1}{n}, 0\right\}$, which is a $C^{2}$ function on $\left[0, h_{0 n}\right]$ for some $h_{0 n}<h_{\infty}$. Now we consider the free boundary problem (1.1) with initial data $u_{0}=v(x)$, and with $u_{0}=v_{n}$. Denote the corresponding free boundaries by $h(t ; v)$ and $h\left(t ; v_{n}\right)$, respectively. By $(2.16)$ we see that there exists $\delta_{1}>0$ such that

$$
h(\infty ; v)-h(0 ; v) \geq 3 \delta_{1}>0 .
$$

Hence for large $n$ we have

$$
h\left(\infty ; v_{n}\right)-h\left(0 ; v_{n}\right) \geq 2 \delta_{1}>0
$$

since $\left\|v_{n}-v\right\|_{C^{2}\left(\left[0, h_{0 n}\right]\right)}=\frac{1}{n}$ is small. Moreover, since $h\left(0 ; v_{n}\right)=h_{0 n} \geq h_{\infty}-\delta_{1} \equiv h(0 ; v)-\delta_{1}$ for large $n$, we have

$$
h\left(\infty ; v_{n}\right) \geq h\left(0 ; v_{n}\right)+2 \delta_{1} \geq h_{\infty}+\delta_{1} .
$$

Choose $\tau$ sufficiently large such that

$$
u(\tau, x) \geq v_{n}(x) \equiv v(x)-\frac{1}{n} \quad \text { for } x \in\left[0, h_{0 n}\right] .
$$


By comparison principle and (2.17) we have

$$
h_{\infty}=h(\infty ; u(\tau, x)) \geq h\left(\infty ; v_{n}\right) \geq h_{\infty}+\delta_{1} .
$$

This contradiction shows that $\omega(u)=\{0\}$.

Finally, we prove $\|u(t, \cdot)\|_{L^{\infty}(I(t))} \rightarrow 0$ as $t \rightarrow \infty$. For any $\varepsilon>0$, define $\delta$ as above. Since $u$ converges to 0 uniformly on $\left[0, h_{\infty}-\delta\right]$ as $t \rightarrow \infty$, there exists $T_{1}>0$ such that

$$
0 \leq u(t, x)<\varepsilon \text { for } x \in\left[0, h_{\infty}-\delta\right] \text { and } t>T_{1} .
$$

Combining this inequality with $(2.15)$ we obtain $\lim _{t \rightarrow \infty}\|u(t, \cdot)\|_{L^{\infty}(I(t))}=0$. This proves the lemma.

Theorem 1.1 can be proved by combining the above lemmas.

\section{Applications}

As applications of the general convergence theorem, in this section we study the asymptotic behavior of solutions to (1.1) with monostbale and combustion types of nonlinearities.

\subsection{Monostable case}

Throughout this subsection, we assume that $f$ is of $\left(f_{M}\right)$ type. In this case, problem (1.4) has only two bounded, nonnegative solutions. One is the Type $I$ solution $v(x) \equiv 0(x \geq 0)$, the other is a Type II solution (denoted by $v^{*}$ ) which satisfies $v^{*}(x)>0, v^{*}(x) \nearrow 1$ as $x \rightarrow \infty$. By Theorem 1.1, we have

$$
\omega(u)=\{0\} \text { or }\left\{v^{*}\right\}
$$

Lemma 3.1. Let $(u, h)$ be any solution of (1.1). If $h_{\infty}<\infty$, then $h_{\infty} \leq \frac{\pi}{\sqrt{f^{\prime}(0)}}$ and $\lim _{t \rightarrow \infty}\|u(t, \cdot)\|_{L^{\infty}(I(t))}=0$.

Proof. Assume, on the contrary, that $h_{\infty}>\frac{\pi}{\sqrt{f^{\prime}(0)}}$. Take any number $l$ satisfying $\max \left\{h_{0}, \frac{\pi}{\sqrt{f^{\prime}(0)}}\right\}<l<$ $h_{\infty}$. Then there exists $T>0$ such that $h(T)=l$ and $u(T, x)>0$ in $(0, l)$.

By a simple phase plane analysis (cf. $[2,5]$ ) we know that, for each $\alpha \in\left(0, \alpha_{0}\right)$ with $\alpha_{0}=$ $\left(2 \int_{0}^{1} f(s) d s\right)^{1 / 2}$, problem

$$
\left\{\begin{array}{l}
q^{\prime \prime}+f(q)=0, \quad x>0 \\
q(0)=0, \quad q^{\prime}(0)=\alpha
\end{array}\right.
$$

has a solution $q_{\alpha}(x)$, which is positive in some interval $\left(0,2 x_{\alpha}\right)$ with

$$
x_{\alpha}=\int_{0}^{q\left(x_{\alpha}\right)}\left[\alpha^{2}-2 F(s)\right]^{-1 / 2} d s,
$$

where $F(u)=\int_{0}^{u} f(s) d s$. Moreover $q_{\alpha}(x)$ is symmetric with respect to $x=x_{\alpha}$ and $q_{\alpha}^{\prime}(x)>0$ for $x \in\left(0, x_{\alpha}\right)$. Denote $q^{\alpha}:=q_{\alpha}\left(x_{\alpha}\right)=\max _{x \in\left[0,2 x_{\alpha}\right]} q_{\alpha}(x)$. It is easily seen that $q^{\alpha}$ is strictly increasing in $\alpha$. In addition, as $\alpha \rightarrow 0+0, q^{\alpha} \rightarrow 0$ and $x_{\alpha} \rightarrow \frac{\pi}{2 \sqrt{f^{\prime}(0)}}<\frac{l}{2}$ by (3.2). Thus we can find $\alpha_{*}>0$ small such that $q_{\alpha_{*}}(0)=q_{\alpha_{*}}\left(2 x_{\alpha_{*}}\right)=0$ and $q_{\alpha_{*}}(x)<u(T, x)$ in $\left(0,2 x_{\alpha_{*}}\right)$. It follows from the comparison principle that

$$
q_{\alpha_{*}}(x) \leq u(t, x) \text { for } t>T, 0<x<2 x_{\alpha_{*}} .
$$

This is a contradiction to Lemma 2.11 and thus $h_{\infty} \leq \frac{\pi}{\sqrt{f^{\prime}(0)}}$. The conclusion $\|u(t, \cdot)\|_{L^{\infty}(I(t))} \rightarrow 0(t \rightarrow$ $\infty)$ follows from Lemma 2.11. This proves the lemma. 
Lemma 3.2. Let $(u, h)$ be any solution of (1.1). If $h_{\infty}=\infty$, then $\lim _{t \rightarrow \infty} u(t, x)=v^{*}(x)$ locally uniformly in $[0, \infty)$.

Proof. Since $h_{\infty}=\infty$, we can take $T>0$ such that $h(T)>\frac{\pi}{\sqrt{f^{\prime}(0)}}$ and $u(T, x)>0$ in $(0, h(T))$. As in the proof of Lemma 3.1, this implies that $\liminf _{t \rightarrow \infty} u(t, x)>0$ in $(0, h(T))$. Therefore, $u \not \rightarrow 0$ and the conclusion follows from Theorem 1.1.

Using Lemmas 3.1 and 3.2 we have the following theorem.

Theorem 3.3 (Dichotomy). Let $f$ be of $\left(\mathrm{f}_{M}\right)$ type, $(u, h)$ be a solution of problem (1.1). Then either

(i) Spreading: $h_{\infty}=\infty$ and $\lim _{t \rightarrow \infty} u(t, x)=v^{*}$ locally uniformly in $[0, \infty)$; or

(ii) Vanishing: $h_{\infty} \leq \frac{\pi}{\sqrt{f^{\prime}(0)}}$ and $\lim _{t \rightarrow \infty} u(t, x)=0$ uniformly in $I(t)$.

Now we give some sufficient conditions for spreading and vanishing.

Lemma 3.4. If $h_{0} \geq \frac{\pi}{\sqrt{f^{\prime}(0)}}$, then $h_{\infty}=\infty$.

Proof. If $h_{\infty}<\infty$, then we have $h_{\infty} \leq \frac{\pi}{\sqrt{f^{\prime}(0)}}$ by Lemma 3.1. However, it follows from $h^{\prime}(t)>0$ and $h_{0} \geq \frac{\pi}{\sqrt{f^{\prime}(0)}}$ that $h_{\infty}>\frac{\pi}{\sqrt{f^{\prime}(0)}}$. This is a contradiction.

Lemma 3.5. Assume that $h_{0}<\frac{\pi}{\sqrt{f^{\prime}(0)}}$. Then $h_{\infty}=\infty$ if

$$
\mu \geq \mu_{1}:=\left(1+\left\|u_{0}\right\|_{L^{\infty}\left(\left[0, h_{0}\right]\right)}\right)\left(2 \int_{0}^{h_{0}} x u_{0}(x) d x\right)^{-1} \frac{\pi^{2}}{f^{\prime}(0)} .
$$

Proof. The proof is divided into two cases: (a) $\left\|u_{0}\right\|_{L^{\infty}\left(\left[0, h_{0}\right]\right)} \leq 1$ and (b) $\left\|u_{0}\right\|_{L^{\infty}\left(\left[0, h_{0}\right]\right)}>1$.

We first consider case (a) and to derive a contradiction by assuming that $h_{\infty}<\infty$. In this case $0<u(t, x) \leq 1$ for $t>0$ and $x \in(0, h(t))$ by the comparison principle, and $u \rightarrow 0$ as $t \rightarrow \infty$ by Lemma 3.1. A direct calculation gives

$$
\begin{aligned}
\frac{d}{d t} \int_{0}^{h(t)} x u(t, x) d x & =\int_{0}^{h(t)} x u_{t}(t, x) d x=\int_{0}^{h(t)} x\left[u_{x x}+f(u)\right] d x \\
& =-\frac{1}{\mu} h h^{\prime}+u(t, 0)+\int_{0}^{h(t)} x f(u) d x .
\end{aligned}
$$

Integrating it from 0 to $t$ gives

$$
\int_{0}^{h(t)} x u(t, x) d x-\int_{0}^{h_{0}} x u_{0}(x) d x \geq \frac{1}{2 \mu} h_{0}^{2}-\frac{1}{2 \mu} h^{2}(t) .
$$

Letting $t \rightarrow \infty$ we have

$$
-\int_{0}^{h_{0}} x u_{0}(x) d x \geq \frac{1}{2 \mu}\left(h_{0}^{2}-h_{\infty}^{2}\right)>-\frac{\pi^{2}}{2 \mu f^{\prime}(0)}
$$

by Lemma 3.1. This contradicts the definition of $\mu_{1}$ and the choice of $\mu \geq \mu_{1}$.

For the case $\left\|u_{0}\right\|_{L^{\infty}\left(\left[0, h_{0}\right]\right)}>1$, we take $\underline{u}_{0}(x)=\frac{u_{0}(x)}{\left\|u_{0}\right\|_{L^{\infty}\left(\left[0, h_{0}\right]\right)}}$. Let $(\underline{u}, \underline{h})$ be the solution of (1.1) with initial data $\underline{u}(0, x)=\underline{u}_{0}(x)$. Then Lemma 2.1 implies $\underline{h}(t) \leq h(t)$ for $t>0$. Note $\left\|\underline{u}_{0}\right\|_{L^{\infty}\left(\left[0, h_{0}\right]\right)}=1$, we can apply the result in case (a) to show that $\lim _{t \rightarrow \infty} \underline{h}(t)=\infty$ if $\mu \geq \mu_{1}$. Thus we also have $h_{\infty}=\infty$. This completes the proof. 
Lemma 3.6. Suppose that $h_{0}<\frac{\pi}{2 \sqrt{f^{\prime}(0)}}$ and $\left\|u_{0}\right\|_{L^{\infty}\left(\left[0, h_{0}\right]\right)}$ is sufficiently small. Then there exists $\mu_{0}>0$ depending on $u_{0}, h_{0}$ and $f$ such that $h_{\infty}<\infty$ if $\mu \leq \mu_{0}$.

Proof. Since $h_{0}<\frac{\pi}{2 \sqrt{f^{\prime}(0)}}$, there exists a small $\delta>0$ such that

$$
\frac{\pi^{2}}{4(1+\delta)^{2} h_{0}^{2}}-f^{\prime}(0) \geq 2 \delta
$$

There exists $s>0$ sufficiently small such that

$$
f(u) \leq\left(f^{\prime}(0)+\delta\right) u \quad \text { for } 0 \leq u \leq s .
$$

Define

$$
k(t):=h_{0}\left(1+\delta-\frac{\delta}{2} e^{-\delta t}\right) \quad \text { and } \quad w(t, x):=s e^{-\delta t} \cdot \cos \frac{\pi x}{2 k(t)} .
$$

A direct calculation shows that $(w, k)$ is an upper solution of $(1.1)$ and $h(t) \leq k(t) \leq h_{0}(1+\delta)$ provided

$$
0<\mu<\mu_{0}:=\frac{\delta^{2} h_{0}^{2}}{\pi s} \quad \text { and } \quad\left\|u_{0}\right\|_{L^{\infty}\left(\left[0, h_{0}\right]\right)} \leq s \cos \frac{\pi}{2+\delta} .
$$

This proves the lemma.

The following theorem shows the existence of a sharp threshold $\mu^{*}>0$ which separates the spreading and vanishing for the case $h_{0}<\frac{\pi}{2 \sqrt{f^{\prime}(0)}}$. To emphasize the dependence of $u$ and $h$ on $\mu$, we write $u, h(t)$ and $h_{\infty}$ as $u^{\mu}, h^{\mu}(t)$ and $h_{\infty}^{\mu}$ in the following theorem.

Theorem 3.7 (Sharp threshold of $\mu$ ). Assume that $h_{0}<\frac{\pi}{\sqrt{f^{\prime}(0)}}$. Then there exists $\mu^{*} \geq 0$ depending on $u_{0}, h_{0}$ and $f$ such that $h_{\infty} \leq \frac{\pi}{\sqrt{f^{\prime}(0)}}$ if $\mu \leq \mu^{*}$, and $h_{\infty}=\infty$ if $\mu>\mu^{*}$.

Moreover, if $h_{0}<\frac{\pi}{2 \sqrt{f^{\prime}(0)}}$ and if $\left\|u_{0}\right\|_{L^{\infty}\left(\left[0, h_{0}\right]\right)}$ is sufficiently small, then $\mu^{*}>0$.

Proof. Define

$$
\Sigma_{1}:=\left\{\mu>0: h_{\infty}^{\mu}=\infty\right\} .
$$

By Lemma 3.5 we have $\left[\mu_{1}, \infty\right) \subset \Sigma_{1}$. It is easily seen by the comparison principle that $\Sigma_{1}$ is an interval of the form $\left[\mu^{*}, \infty\right)$ or $\left(\mu^{*}, \infty\right)$, where $\mu^{*}:=\inf \Sigma_{1} \leq \mu_{1}$.

If $\mu^{*}=0$, then there is nothing left to prove. We now assume that $\mu^{*}>0$ and to show that $\mu^{*} \notin \Sigma_{1}$. If $\mu^{*} \in \Sigma_{1}$, then we can find $T>0$ such that $h^{\mu^{*}}(T)>\frac{\pi}{\sqrt{f^{\prime}(0)}}$. Since $\left(u^{\mu}, h^{\mu}\right)$ depends continuously on $\mu$, there exists $\delta>0$ small such that $h^{\mu}(T)>\frac{\pi}{\sqrt{f^{\prime}(0)}}$ for $\mu \in\left[\mu^{*}-\delta, \mu^{*}\right]$. Therefore $h_{\infty}^{\mu}=\infty$ by Lemma 3.4. This implies that $\left[\mu^{*}-\delta, \mu^{*}\right] \subset \Sigma_{1}$ and so inf $\Sigma_{1} \leq \mu^{*}-\delta$, contradicting the definition of $\mu^{*}$. Hence $\mu^{*} \notin \Sigma_{1}$.

When $h_{0}<\frac{\pi}{2 \sqrt{f^{\prime}(0)}}$ and $\left\|u_{0}\right\|_{L^{\infty}\left(\left[0, h_{0}\right]\right)}$ is sufficiently small, Lemma 3.6 implies that $\left[0, \mu^{*}\right]$ is not a singleton $\{0\}$. Therefore, $\mu^{*}>0$.

Next we give a sharp threshold of the size of the initial data which separates the spreading and vanishing.

Theorem 3.8 (Sharp threshold of the initial data). Suppose that $\phi \in \mathscr{X}\left(h_{0}\right)$ for some $h_{0}>0$. Let $(u, h)$ be a solution of (1.1) with $u_{0}=\lambda \phi$ for some $\lambda \geq 0$. Then there exists $\lambda^{*} \in[0, \infty]$ depending on $h_{0}, \phi$ and $f$ such that spreading happens when $\lambda>\lambda^{*}$ and vanishing happens when $0 \leq \lambda \leq \lambda^{*}$. 
Proof. To emphasize the dependence of the solution $(u, h)$ on the parameter $\lambda$, we write $\left(u_{\lambda}, h_{\lambda}\right)$ instead of $(u, h)$. Define

$$
\Sigma_{0}:=\left\{\lambda>0: u_{\lambda}(t, x) \rightarrow 0 \text { as } t \rightarrow \infty \text { uniformly in } I(t)\right\}
$$

and $\lambda^{*}:=\sup \Sigma_{0}$. When $\Sigma_{0}=\emptyset$, we set $\lambda^{*}=0$. By Lemma 3.4, such a case happens when $h_{0} \geq \frac{\pi}{\sqrt{f^{\prime}(0)}}$. If $\Sigma_{0}=(0, \infty)$, then $\lambda^{*}=\infty$, which means that any $u_{\lambda}$ vanishes no matter how large $\lambda$ is. Note that such a case really happens when $h_{0}$ is sufficiently small and when $-f(u)$ grows sufficiently fast. (Indeed, in [5] the authors proved that any solution of (1.1) with the Robin boundary condition replaced by a Neumann one $u_{x}(t, 0)=0$ converges to 0 if $\liminf _{u \rightarrow \infty}-f(u) / u^{8}>0$ and if the initial support $\left[0, h_{0}\right]$ is narrow enough. They constructed an even upper solution to block the spreading of the solution. We see that their upper solution can also be used to our problem since the Robin boundary condition implies that $\left.u_{x}(t, 0) \geq 0\right)$.

In what follows we consider the case where $\Sigma_{0} \neq \emptyset$ and $\Sigma_{0} \neq(0, \infty)$. Then, by the comparison principle, it is easily seen that $\Sigma_{0}$ is an interval $\left(0, \lambda^{*}\right)$ or $\left(0, \lambda^{*}\right]$ for some $\lambda^{*} \in(0, \infty)$. This only happens when $h_{0}<\frac{\pi}{\sqrt{f^{\prime}(0)}}$.

Assume that $\lambda^{*} \notin \Sigma_{0}$. Then $u_{\lambda^{*}}(t, x) \rightarrow v^{*}$ as $t \rightarrow \infty$, locally uniformly in $[0, \infty)$ by Theorem 3.3 . Hence $h_{\lambda^{*}}(T)>\frac{\pi}{\sqrt{f^{\prime}(0)}}$ for some $T>0$. Since $h_{\lambda}$ depends continuously on its initial value $\lambda \phi$, we have $h_{\lambda^{*}-\varepsilon}(T)>\frac{\pi}{\sqrt{f^{\prime}(0)}}$ for any small $\varepsilon>0$. So $\lambda^{*}-\varepsilon \notin \Sigma_{0}$ by Theorem 3.3 and Lemma 3.4. This, however, contradicts the definition of $\lambda^{*}$ and the fact that $\Sigma_{0}$ is an interval by the comparison principle. Therefore, $\lambda^{*} \in \Sigma_{0}$ and $\Sigma_{0}=\left(0, \lambda^{*}\right]$. The proof is completed.

\subsection{Combustion case}

In this subsection, we assume that $f$ is of $\left(\mathrm{f}_{C}\right)$ type. Similar to the monostable case, problem (1.4) now has exactly two bounded, nonnegative solutions: Type $I$ solution $v(x) \equiv 0$ and a Type II solution $v^{*}$ satisfying $v^{*}(x)>0, v^{*}(x) \nearrow 1$ as $x \rightarrow \infty$. By Theorem 1.1, we have the following result.

Theorem 3.9 (Dichotomy). Let $f$ be of $\left(f_{C}\right)$ type and $(u, h)$ be any solution of problem (1.1). Then either

(i) Spreading: $h_{\infty}=\infty$ and $\lim _{t \rightarrow \infty} u(t, x)=v^{*}$ locally uniformly in $[0, \infty)$; or,

(ii) Vanishing: $\lim _{t \rightarrow \infty} u(t, x)=0$ locally uniformly in $I_{\infty}$.

Lemma 3.10. Suppose that $u_{0} \in \mathscr{X}\left(h_{0}\right)$ for some $h_{0}>0$. If $\left\|u_{0}\right\|_{L^{\infty}\left(\left[0, h_{0}\right]\right)} \leq \theta$, then $h_{\infty}<\infty$, and so $u(t, \cdot) \rightarrow 0(t \rightarrow \infty)$ in $L^{\infty}((I(t))$ topology.

Proof. By the comparison principle we have $u(t, x) \leq \theta$ for $t \geq 0$. Thus the equation we are dealing with reduces to the heat equation $u_{t}=u_{x x}$. Consider the Cauchy problem

$$
\left\{\begin{array}{l}
w_{t}-w_{x x}=0, \quad t>0, x \in \mathbb{R} \\
w(0, x)=\Phi(x), x \in \mathbb{R}
\end{array}\right.
$$

where

$$
\Phi(x)= \begin{cases}u_{0}(x), & x \in\left[0, h_{0}\right), \\ u_{0}(-x), & x \in\left(-h_{0}, 0\right), \\ 0, & \text { otherwise }\end{cases}
$$

Then

$$
w(t, x)=\frac{1}{2 \sqrt{\pi t}} \int_{\mathbb{R}} e^{-\frac{(x-\xi)^{2}}{4 t}} \Phi(\xi) d \xi \leq \frac{1}{\sqrt{\pi t}} \int_{0}^{h_{0}} u_{0}(\xi) d \xi \leq \frac{\theta h_{0}}{\sqrt{\pi t}} .
$$

Since $w(t, 0)-b w_{x}(t, 0)=w(t, 0)>0$, we have $u(t, x) \leq w(t, x)$ for $t>0$ and $0<x \leq h(t)$ by the comparison principle. Therefore, there exists $T>0$ such that

$$
\max _{0 \leq x \leq h(T)} u(T, x) \leq \max _{0 \leq x \leq h(T)} w(T, x) \leq \frac{\theta h_{0}}{\sqrt{\pi T}} \leq \eta:=\frac{1}{2} \min \left\{\theta, \frac{\pi}{8 \mu}\right\} .
$$


Choose $l>h(T)$ such that

$$
u(T, x)<2 \eta \cos \frac{\pi x}{2 l} \quad \text { for } x \in I(T) .
$$

Define

$$
k(t):=l\left(2-e^{-\frac{\pi^{2}}{16 l^{2}} t}\right) \in(l, 2 l) \quad \text { and } \quad \zeta(t, x):=2 \eta e^{-\frac{\pi^{2}}{16 l^{2}} t} \cos \frac{\pi x}{2 k(t)} .
$$

A direct calculation shows that $(\zeta, k)$ is an upper solution of (1.1). Applying Lemma 2.1 we can obtain $h(t) \leq k(t)$ for $t \geq T$. This implies $h_{\infty}<2 l<\infty$ and the conclusion follows from Lemma 2.11.

Theorem 3.11 (Sharp threshold of the initial data). Suppose that $\phi \in \mathscr{X}\left(h_{0}\right)$ for some $h_{0}>0$, $\left(u_{\lambda}, h_{\lambda}\right)$ is the solution of (1.1) with $u_{0}=\lambda \phi$ for some $\lambda>0$. Then there exists $\lambda^{*} \in(0, \infty]$ depending on $h_{0}, \phi$ and $f$ such that spreading happens when $\lambda>\lambda^{*}$ and vanishing happens when $0<\lambda \leq \lambda^{*}$.

Proof. Denote $h_{\lambda, \infty}:=\lim _{t \rightarrow \infty} h_{\lambda}(t)$ and define

$$
\Sigma_{0}:=\left\{\lambda>0: u_{\lambda}(t, x) \rightarrow 0 \text { as } t \rightarrow \infty \text { locally uniformly in }\left[0, h_{\lambda, \infty}\right)\right\} .
$$

Lemma 3.10 implies that $\Sigma_{0} \neq \emptyset$. The comparison principle implies that $\Sigma_{0}$ is an interval of the form $\left(0, \lambda^{*}\right)$ or $\left(0, \lambda^{*}\right]$, where $\lambda^{*}:=\sup \Sigma_{0}$.

If $\lambda^{*}=\infty$, then $\Sigma_{0}=(0, \infty)$ and there is nothing left to prove. By [5, Proposition 5.12], such a case really happens when $h_{0}$ is sufficiently small and when $-f(u)$ grows very fast.

In what follows we consider the case $0<\lambda^{*}<\infty$. We will show that $\lambda^{*} \in \Sigma_{0}$.

As in the proof of Lemma 3.1 (see also $[2,5]$ ), for each $\alpha \in\left(0, \alpha_{0}\right)$ with $\alpha_{0}:=\left(2 \int_{0}^{1} f(s) d s\right)^{1 / 2}$, problem

$$
\left\{\begin{array}{l}
q^{\prime \prime}+f(q)=0, \quad x>0 \\
q(0)=0, q^{\prime}(0)=\alpha
\end{array}\right.
$$

has a solution $q_{\alpha}(x)$. Multiplying the equation by $q^{\prime}$ and integrating it on $[0, x]$, one obtains the following conclusions. The maximum value $q^{\alpha}$ of $q_{\alpha}(x)$ is attained at some point $x_{\alpha}, q_{\alpha}(x)$ is positive in $\left(0,2 x_{\alpha}\right)$ and is symmetric with respect to $x=x_{\alpha}$. Moreover,

$$
\alpha^{2}=2 \int_{0}^{q^{\alpha}} f(s) d s \quad \text { and } \quad x_{\alpha}=\int_{0}^{q^{\alpha}}\left(2 \int_{r}^{q^{\alpha}} f(s) d s\right)^{-1 / 2} d r .
$$

In addition, $q^{\alpha} \rightarrow \theta$ as $\alpha \rightarrow 0, q^{\alpha} \rightarrow 1$ as $\alpha \rightarrow \alpha_{0}$. Define

$$
H:=\inf _{0<\alpha<\alpha_{0}} x_{\alpha}=\inf _{\theta<q<1} \int_{0}^{q}\left(2 \int_{r}^{q} f(s) d s\right)^{-1 / 2} d r .
$$

Then for any interval $\left[x_{1}, x_{2}\right]$ with width $x_{2}-x_{1}>2 H$, there exists $\alpha \in\left(0, \alpha_{0}\right)$ such that $q_{\alpha}\left(x-x_{1}\right)$ is a solution of

$$
v^{\prime \prime}+f(v)=0 \quad\left(x_{1}<x<x_{2}\right), \quad v(x)>0 \text { in }\left(x_{1}, x_{2}\right), \quad v\left(x_{1}\right)=v\left(x_{2}\right)=0,
$$

and $\max _{x \in\left[x_{1}, x_{2}\right]} v(x)=q^{\alpha} \in(\theta, 1)$.

Let $v^{*}$ be the unique Type II positive solution of (1.4) as stated in Theorem 3.9. Since $v^{*}(x) \nearrow 1(x \rightarrow$ $\infty)$, there exists $z^{*}>0$ such that $v^{*}(x)>q^{\alpha}\left(x>z^{*}\right)$ for some $\alpha \in\left(0, \alpha_{0}\right)$. Hence

$$
v^{*}(x)>q^{\alpha} \geq q_{\alpha}\left(x-z^{*}\right) \quad \text { for } \quad x \in\left[z^{*}, z^{*}+2 x_{\alpha}\right] .
$$

Suppose that $\lambda^{*} \notin \Sigma_{0}$. Then, by Theorem $3.9, u_{\lambda^{*}}(t, \cdot)$ converges locally uniformly to $v^{*}(x)$ as $t \rightarrow \infty$. Therefore, for some large $T>0$ we have

$$
u_{\lambda^{*}}(T, x)>q_{\alpha}\left(x-z^{*}\right), \quad x \in\left[z^{*}, z^{*}+2 x_{\alpha}\right] .
$$


Note that $u_{\lambda}$ depends on $\lambda$ continuously. So we have

$$
u_{\lambda^{*}-\varepsilon}(T, x) \geq q_{\alpha}\left(x-z^{*}\right), \quad x \in\left[z^{*}, z^{*}+2 x_{\alpha}\right]
$$

for any small $\varepsilon>0$. This implies that $u_{\lambda^{*}-\varepsilon} \nrightarrow>0$ since $q_{\alpha}\left(x-z^{*}\right)$ is a stationary solution of $v^{\prime \prime}+f(v)=0$. Therefore $\lambda^{*}-\varepsilon \notin \Sigma_{0}$. This contradicts the definitions of $\Sigma_{0}$ and $\lambda^{*}$.

Remark 3.12. In Theorem 3.9 (ii) we obtain $u \rightarrow 0$ locally uniformly in $I_{\infty}$. The following comment explains why we can not obtain a similar uniform convergence as in Theorem 3.3 (ii). Consider the problem (1.1) in $\left(\mathrm{f}_{C}\right)$ case. Assume that the initial data is $\lambda \phi$ for some given $\phi \in \mathscr{X}\left(h_{0}\right)\left(h_{0}>0\right.$ is large). Define

$$
\Sigma^{0}:=\left\{\lambda>0: u_{\lambda} \rightarrow 0 \text { uniformly in } I(t)\right\}
$$

and

$$
\Sigma^{1}:=\left\{\lambda>0: u_{\lambda} \rightarrow v^{*} \text { locally uniformly in }[0, \infty)\right\} .
$$

From the proof of Theorem 3.11 we see that $\Sigma^{1}$ is a nonempty open set. On the other hand, Lemma 3.10 implies that $\Sigma^{0}$ is also a nonempty open set. Hence $\tilde{\Sigma}:=(0, \infty) \backslash\left(\Sigma^{0} \cup \Sigma^{1}\right) \neq \emptyset$. For each $\tilde{\lambda} \in \tilde{\Sigma}^{*}$, $u_{\tilde{\lambda}}$ must converges to 0 locally uniformly in $[0, \infty)$, but not uniformly in $I(t)$. Therefore, $h_{\tilde{\lambda}, \infty}=\infty$ by Lemma 2.11.

Acknowledgements. This research was partly supported by NSFC (No. 11271285) and by the Fundamental Research Funds for the Central Universities (Tongji University).

\section{References}

[1] S. B. Angenent. The zero set of a solution of a parabolic equation. J. Reine Angew. Math., 390 (1988), 79-96.

[2] D. G. Aronson, H. F. Weinberger. Multidimensional nonlinear diffusion arising in population genetics. Adv. in Math., 30 (1978), 33-76.

[3] G. Bunting, Y. Du, K. Krakowski. Spreading speed revisited: Analysis of a free boundary model. Netw. Heterog. Media., (to appear).

[4] Y. Du, Z. G. Lin. Spreading-vanishing dichtomy in the diffusive logistic model with a free boundary. SIAM J. Math. Anal., 42 (2010), 377-405.

[5] Y. Du, B. D. Lou. Spreading and vanishing in nonlinear diffusion problems with free boundaries. Preprint.

[6] Y. Du, H. Matano. Convergence and sharp thresholds for propagation in nonlinear diffusion problems. J. Eur. Math. Soc., 12 (2010), 279-312.

[7] Y. Kaneko, Y. Yamada. A free boundary problem for a reaction-diffusion equation appearing in ecology. Adv. Math. Sci. Appl., 21 (2011), 467-492.

[8] Z. G. Lin. A free boundary problem for a predator-prey model. Nonlinearity, 20 (2007), 1883-1892. 